\title{
On-sky demonstration of the GMT dispersed fringe phasing sensor prototype on the Magellan telescope
}

\author{
Derek Kopon $^{* a}$, Brian McLeod ${ }^{\mathrm{a}}$, Marcos A. van Dam ${ }^{\mathrm{b}}$, Antonin Bouchez ${ }^{\mathrm{c}}$, Ken McCracken ${ }^{\mathrm{a}}$, Daniel \\ Catropa $^{\mathrm{a}}$, William Podgorski ${ }^{\mathrm{a}}$, Stuart McMuldroch ${ }^{\mathrm{a}}$, Alan Conder ${ }^{\mathrm{c}}$, Laird Close ${ }^{\mathrm{d}}$, Jared Males ${ }^{\mathrm{d}}$, Katie \\ Morzinski $^{\mathrm{d}}$, Timothy Norton ${ }^{\mathrm{a}}$ \\ ${ }^{a}$ Harvard Smithsonian Center for Astrophysics, 60 Garden Street, MA, 02138 \\ ${ }^{\mathrm{b}}$ Flat Wavefronts, 21 Lascelles St., Christchurch 8022, New Zealand \\ ${ }^{\mathrm{c}}$ GMTO Corporation, 251 S. Lake Ave., Suite 300, Pasadena, CA, 91101 \\ ${ }^{\mathrm{d}}$ Steward Observatory, University of Arizona, Tucson, AZ 85721
}

\begin{abstract}
The GMT is an aplanatic Gregorian telescope consisting of 7 primary and secondary mirror segments that must be phased to within a fraction of an imaging wavelength to allow the 25.4 meter telescope to reach its diffraction limit. When operating in Laser Tomographic Adaptive Optics (LTAO) mode, on-axis guide stars will not be available for segment phasing. In this mode, the GMT's Acquisition, Guiding, and Wavefront Sensing system (AGWS) will deploy four pickoff probes to acquire natural guide stars in a 6-10 arcmin annular FOV for guiding, active optics, and segment phasing. The phasing sensor will be able to measure piston phase differences between the seven primary/secondary pairs of up to 50 microns with an accuracy of $50 \mathrm{~nm}$ using a J-band dispersed fringe sensor. To test the dispersed fringe sensor design and validate the performance models, SAO has built and commissioned a prototype phasing sensor on the Magellan Clay 6.5 meter telescope. This prototype uses an aperture mask to overlay 6 GMT-sized segment gap patterns on the Magellan 6.5 meter primary mirror reimaged pupil. The six diffraction patterns created by these subaperture pairs are then imaged with a lenslet array and dispersed with a grism. An on-board phase shifter has the ability to simulate an arbitrary phase shift within subaperture pairs. The prototype operates both on-axis and 6 arcmin off-axis either with AO correction from the Magellan adaptive secondary MagAO system on or off in order to replicate as closely as possible the conditions expected at the GMT.
\end{abstract}

Keywords: Active optics, adaptive optics, Giant Magellan Telescope, phasing, dispersed fringe sensor

\section{INTRODUCTION}

The Giant Magellan Telescope (GMT) is a 25.4 meter diameter telescope whose primary mirror consists of seven 8.4 meter segments, each of which corresponds to a critically sized 1 meter diameter secondary mirror segment. In order for the telescope to reach its diffraction limit, all of the primary-secondary pairs must be phased to within a fraction of an observing wavelength ${ }^{1}$. The phasing of the GMT is uniquely difficult for two reasons ${ }^{2}$. The first is that the doublysegmented primary-secondary design creates degeneracies between segment tip/tilt and piston when measured with offaxis stars ${ }^{3}$. Breaking this degeneracy requires making phasing measurements with multiple off-axis stars simultaneously. The second is the large gap between primary segments: $295 \mathrm{~mm}$ between outer segments and $359 \mathrm{~mm}$ between inner and outer segments, on-axis. These gaps grow larger in the telescope pupil for off-axis stars, because the secondary segments are critically sized to those of the primary and vignette off-axis. These large gaps (compared to inter-segment gaps of $\sim 3 \mathrm{~mm}$ for Keck and JWST ${ }^{4}$ ) require an IR phasing sensor in order to have a wavelength of light long enough to maintain phase coherence ${ }^{5}$. Recent simulations by van Dam ${ }^{6}$ show that a J-band sensor provides the best sky coverage: $90 \%$ at the South Galactic Pole for phase errors below $50 \mathrm{~nm}$ and guide stars as faint as $\mathrm{m}_{\mathrm{J}}=13.6$.

Telescope phase sensing will be one of the many tasks performed by the Active optics, Guiding, and Wavefront Sensor (AGWS) being designed and built by the Smithsonian Astrophysical Observatory ${ }^{7}$ (Fig. 1). The AGWS consists of four

*derek.kopon@cfa.harvard.edu

Adaptive Optics Systems V, edited by Enrico Marchetti, Laird M. Close, Jean-Pierre Véran, Proc. of SPIE Vol. 9909,990946 (c) 2016 SPIE $\cdot$ CCC code: 0277-786X/16/\$18 - doi: 10.1117/12.2232942 
identical movable star probes located in a plane just before the telescope focal plane. Each star probe will contain five selectable optical channels: four visible and one IR. The four visible channels are: a 30 arcsec FOV acquisition camera; a Shack-Hartmann wavefront sensor capable both of long time-scale active optics measurements and ground-layer wavefront sensing at $100 \mathrm{~Hz}$; a fast $(\sim 1 \mathrm{kHz})$ guide channel for natural guide star tip/tilt measurement during LTAO; and a 7-subaperture channel for segment tip/tilt sensing. The IR channel is the J-band phasing sensor that will be fed by a dichroic beamsplitter.

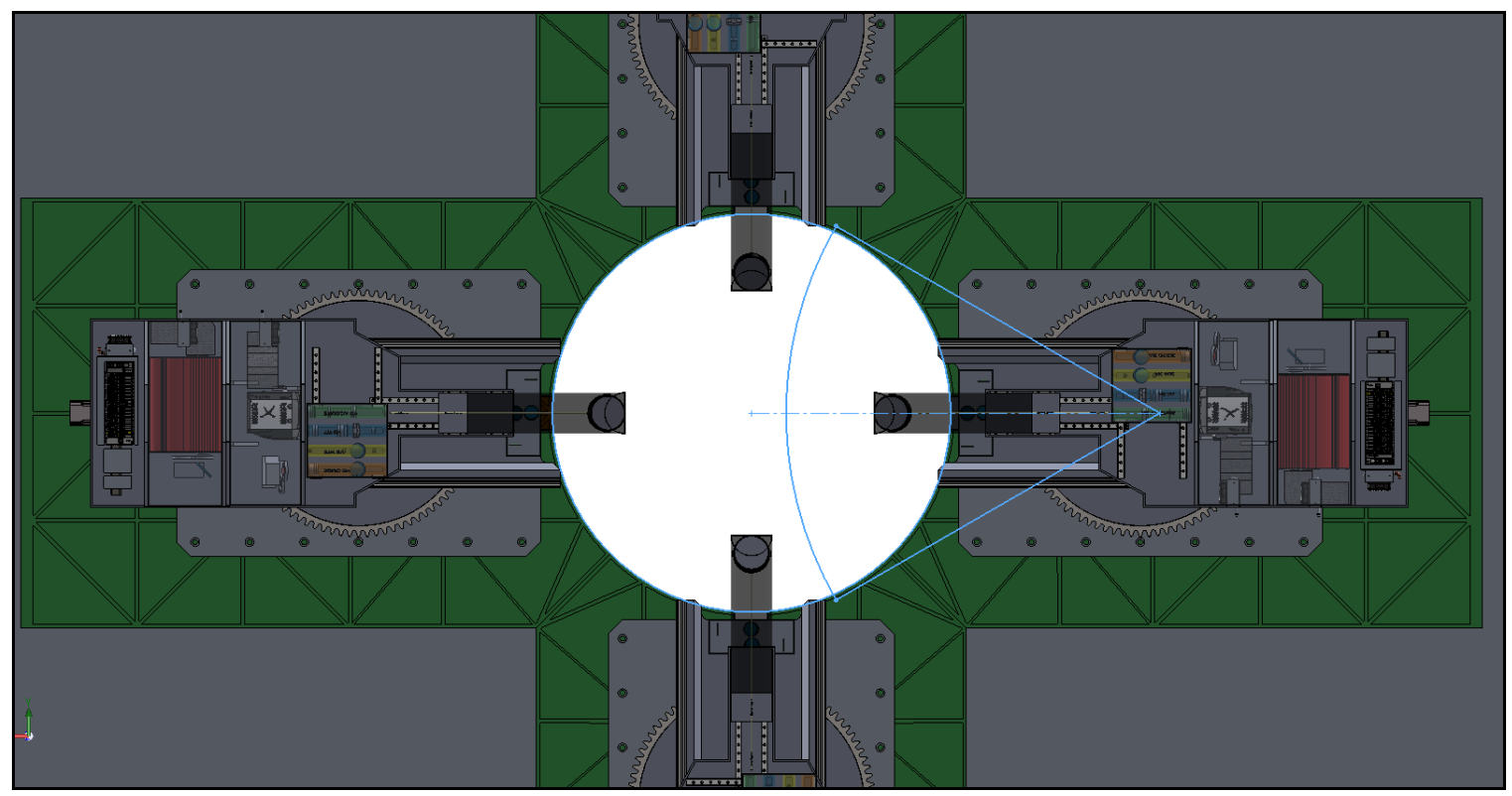

Figure 1: The GMT AGWS as viewed from the telescope direct Gregorian science focal plane looking towards the secondary mirror. The four identical movable star probes will provide target acquisition, active optics sensing, GLAO sensing at $100 \mathrm{~Hz}$, segment tip/tilt correction, fast tip/tilt guiding, and segment phasing.

In order to develop the technology and mitigate the risk associated with our novel Dispersed Fringe Sensor (DFS) design, SAO has undertaken a series of prototyping efforts at the Magellan telescope. An earlier SAO phasing prototype was tested successfully at the Magellan Telescope in $2012^{8}$. This prototype operated at K-band with a seeing-limited (not AO corrected) beam on-axis and used a fast steering mirror for fringe stabilization. However, the recent advent of sub-electron noise fast readout IR arrays has allowed us to eliminate the cryogenic optics and fast steering mirror from the sensor.

Our second prototype, which is the focus of this paper, operates at both I and $\mathrm{J}$ bands, both on-axis and at 6 arcmin offaxis, and with both an AO corrected beam and a seeing limited beam. The GMT DFS operates by using $1.5 \mathrm{~m}$ square apertures placed over each segment boundary in order to generate a set of fringes analogous to a 2-slit diffraction pattern. A grism or prisms is then used to disperse the fringes in direction perpendicular to the diffraction direction. Because the fringe spacing is wavelength dependent, a phase shift between the two segments will appear as a tilt in the dispersed fringes. This fringe tilt will produce a linear displacement of the vertical position of the peak of the Fourier transform, thereby providing a measurement of piston error (Fig. 2) ${ }^{6}$. In order to simulate this method at the Magellan telescope, we overlay six GMT-size segment boundary apertures on the Magellan telescope pupil (Fig. 3). 


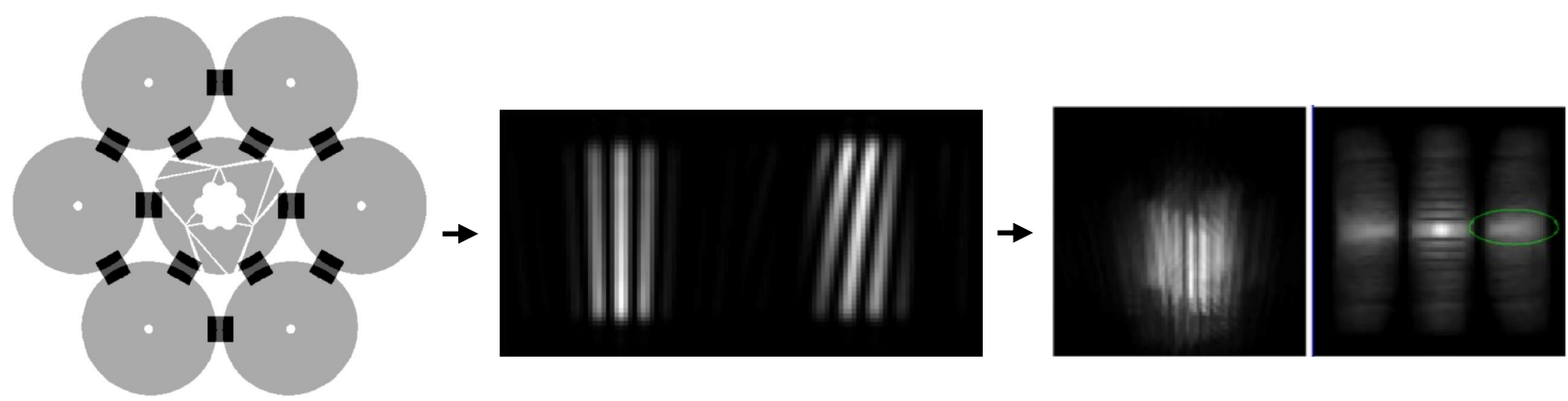

Figure 2: Left: The GMT pupil overlaid with $1.5 \mathrm{~m}$ square apertures at the segment boundaries. Center: Simulated fringes from one subaperture showing 0 piston phase difference (left) and 10 microns (right). Right: Simulated dispersed fringe image and its FFT. The vertical position of the circled peak is a function of the tilt of the fringes and thus the piston error.
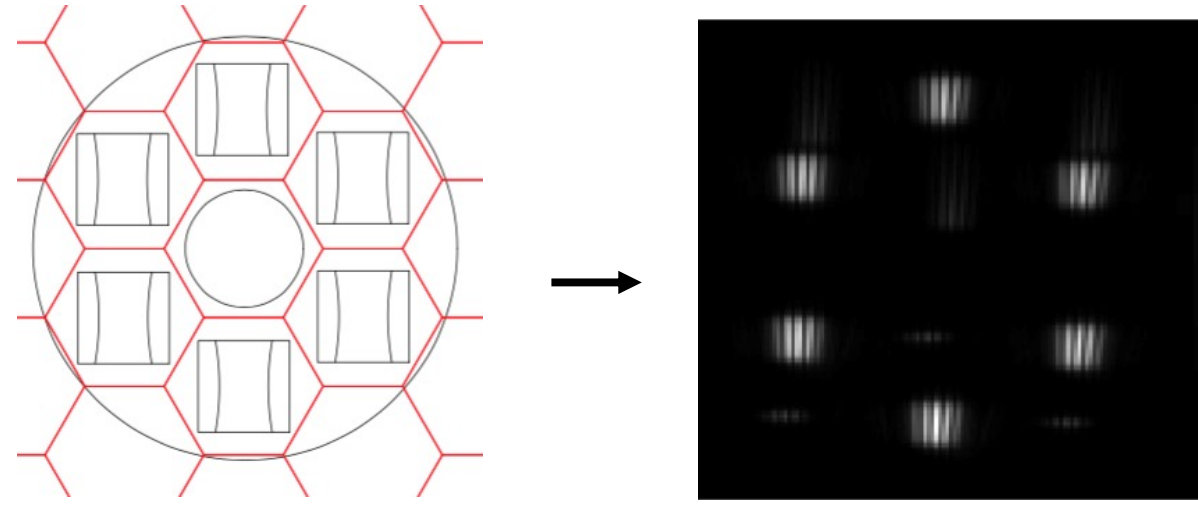

Figure 3: The Magellan pupil overlaid with 6 GMT segment boundary apertures and a hexagonal lenslet array.

\subsection{I and $J$ Band Channels}

In order to maximize sky coverage, the GMT AGWS phasing cameras will operate in J-band using SAPHIRA eAPD arrays because of their fast readout rate and low noise ${ }^{9}$. Because these detectors were not commercially available at the time of the prototype design, we used a 2-channel system that would meet our prototyping goals. The I-band channel uses a fast low read-noise Princeton ProEM512 EMCCD. The J-band channel uses a Raptor Ninox $640 \mathrm{InGaAs}$ detector. The Ninox has lower sensitivity and much higher noise ( $\sim 50$ electrons) than the C-Red. Therefore, the prototype J-band channel is only used on bright stars.

The $\mathrm{I}$ and $\mathrm{J}$ channels are selectable using a manual stage. There is a $\sim 25 \operatorname{arcsec}$ FOV acquisition channel that is fed by a fold flat with a 3 arcsec hole in the center that serves as a field stop for the phasing channels.

\subsection{Optical Design}

Fig. 4 shows the optical design of the I-band channel. Light from the telescope focus is collimated and a pupil image is formed on a laser-cut aperture mask. The mask is followed closely by a hexagonal lenslet array and a grism. An optical relay forms an image of the fringes on the detector. A phase adjuster can be inserted into the beam of the I or J channels in order to simulate a piston phase difference within a subaperture pair. The phase adjuster consists of two plane-parallel pieces of glass: one is fixed, while the other rotates. This rotation changes the path length of the light through the glass and creates a differential phase shift between the beams. Only two subaperture pairs can be phase shifted at a time (usually we used the center top and center bottom pairs). 


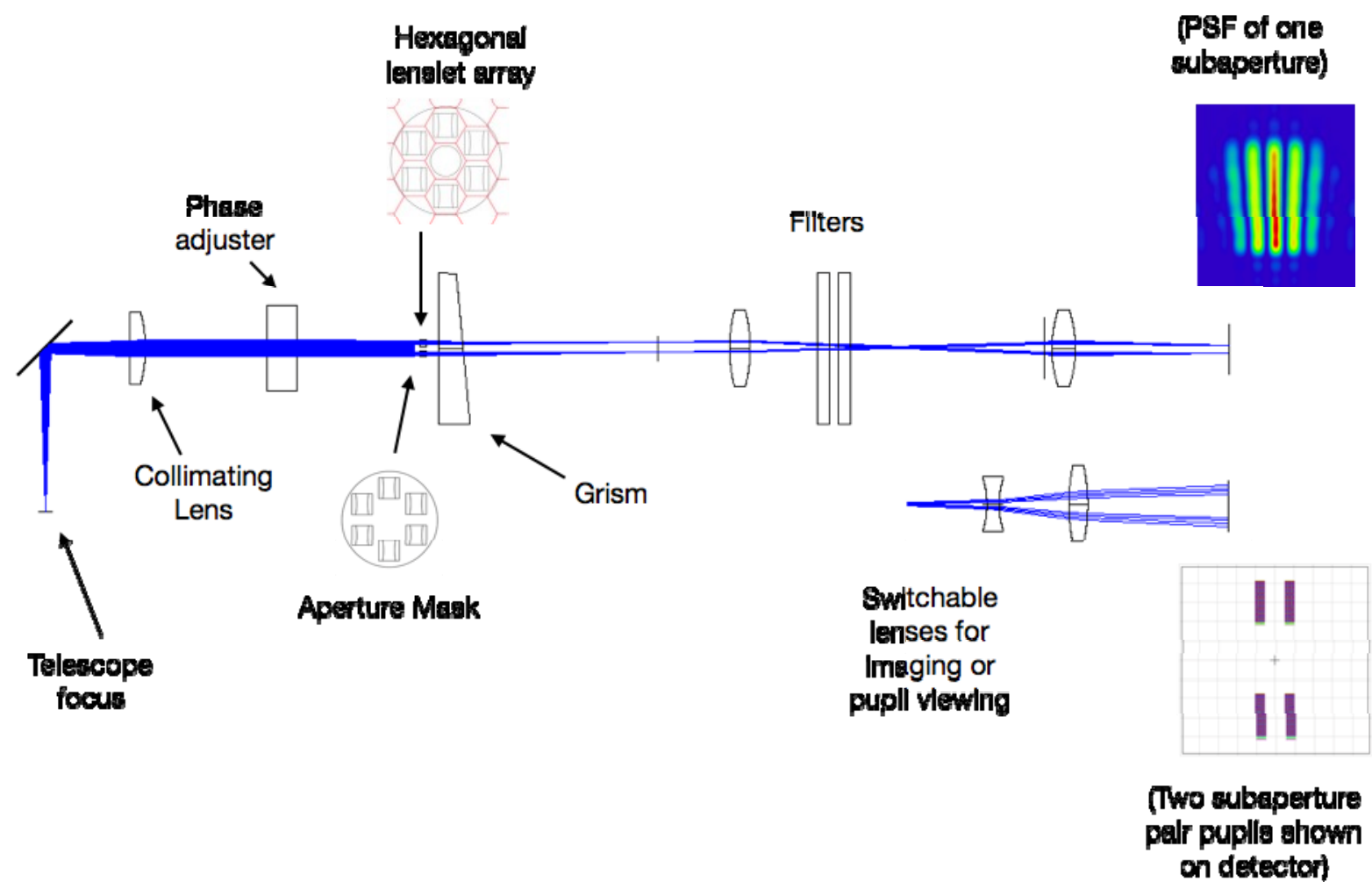

Figure 4: The optical design of the I-band channel, on-axis. Movable stages allow the instrument to operate either in imaging or pupil viewing mode, with or without the phase adjuster.

The Magellan telescopes have significant coma and astigmatism off-axis that must be corrected in order to allow the pupil to be properly imaged and the phase measured. In order to avoid the complexity of trying to optically correct these aberrations over a range of field angles, we decided to statically correct the telescope aberrations at 6 arcmin only with a series of tilted and decentered lenses, since this is within our acquisition range for the AGWS at GMT. These tilted and decentered lenses are contained in the Off-Axis Lens assembly (OAL), which can be inserted into the optical path when the prototype is used off-axis. When the prototype is used on-axis, the OAL is removed. For more details on the prototype optical design, see Kopon et al. $2015^{10}$. 


\section{LABORATORY TESTS}

The prototype was assembled at the CfA and lab tested both there and in the telescope auxiliary building prior to being deployed on the telescope. For our tests we used both an on-board calibration source and a bench-mounted source with a reimaged pupil. Each consisted of a white light source and a pinhole. The bench source allowed us to use a rotating Lexitek phase screen as a turbulence generator in order to operate the prototype under realistic conditions (Fig. 5). The bench source also allowed us to align the optical axis of the prototype to the rotation axis of our mounting plate.

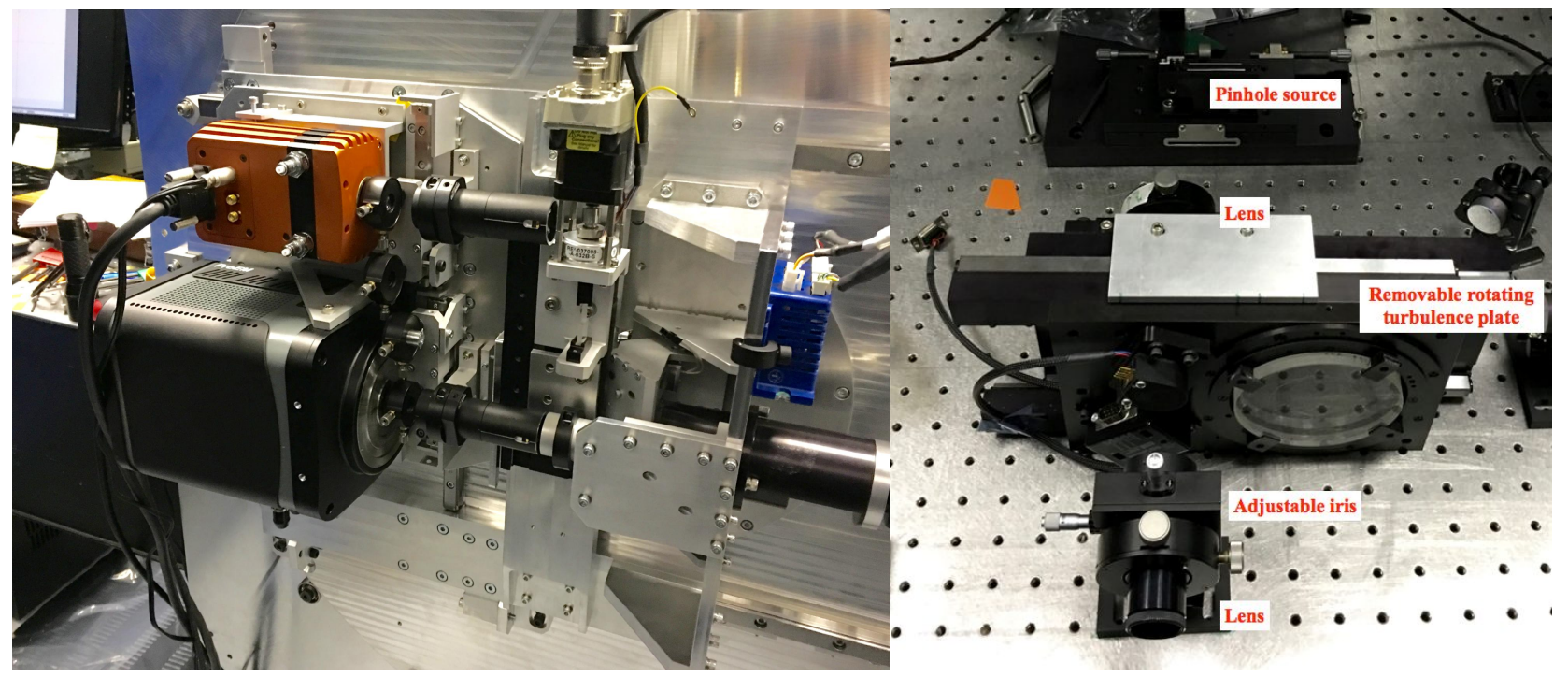

Figure 5: Left: The prototype in the lab. The upper channel is the J-band channel with the Ninox (in orange), the lower channel in the I-band channel with the Princeton EMCCD. Right: The bench calibration source with removable rotating phase plate.

Figure 6 shows a series of 100 ms images, without the phase shifter, added together, background subtracted, and flat fielded $^{11}$. It can be seen that all six sets of fringes are clocked by 3.5 degrees relative to the detector. This clocking is due to the aperture mask being rotated relative to the grism. The tilt can also be seen in the Fourier transform. Additional sets of fringes from the $0^{\text {th }}$ and $2^{\text {nd }}$ orders can also be seen in the image. The fringe contrast is not as good as expected on sky because the point source is resolved. Figure 7 shows the fringes with the phase shifter in the beam.

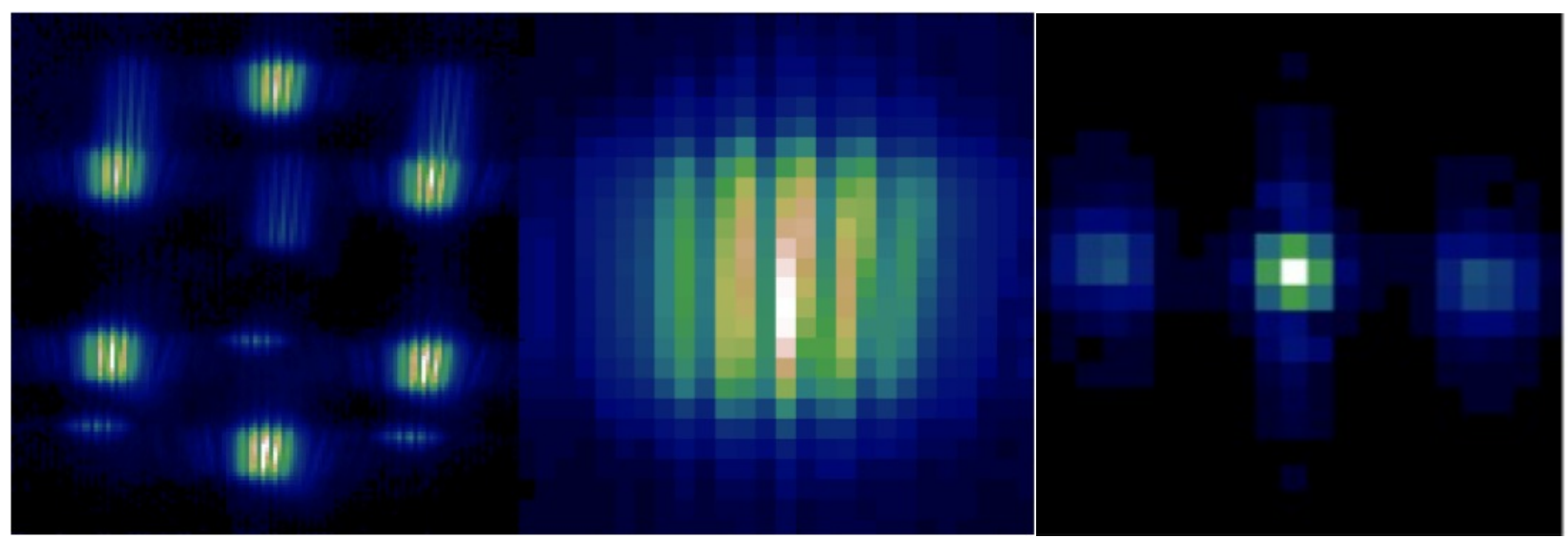

Figure 6: Left: I-band images taken in the lab. Center: A close up of the upper set of fringes. Right: The absolute value of the Fourier transform of the top measured image. 


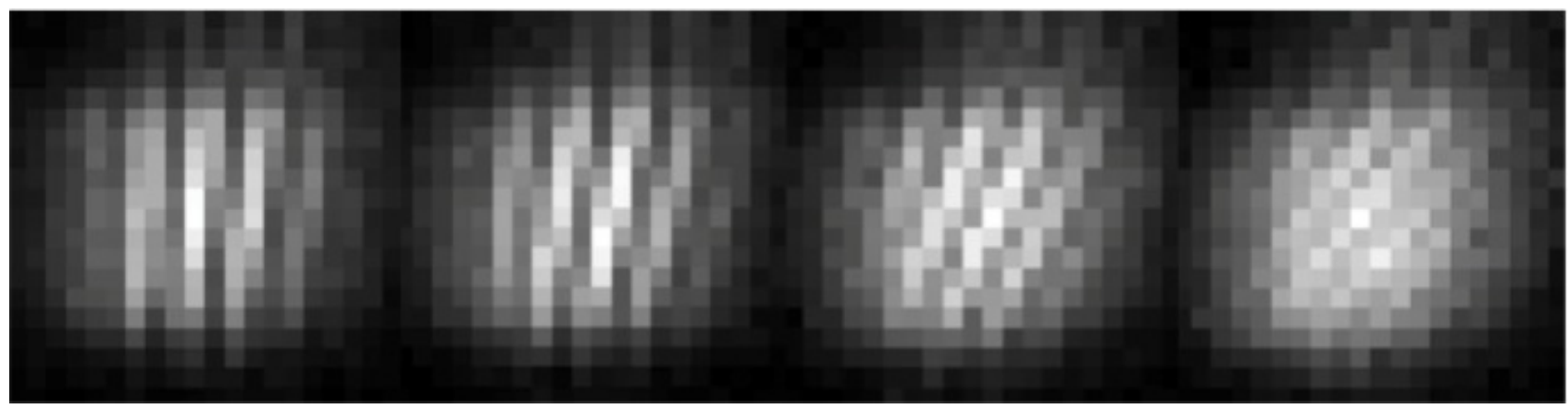

Figure 7: Phase shifted fringes in increments of 3 degrees of the phase adjuster clocking angle, ranging from 22-31 degrees.

It was also observed in the lab (and later verified in Zemax) that lateral chromatic aberration in the relay optics downstream from the grism creates phase biases in the six sets of fringes. Because of the geometry of the lateral color relative to the dispersion direction set by the grism, the effect of this aberration is different in each subaperture. In the four side fringes (two on the left, two on the right), the lateral color results in an additional fringe tilt that adds to the effect of the rotated grism and results in a phase bias. For the top and bottom sets of fringes, since the dispersion direction of the fringes is aligned with the direction of the lateral color, the result is either an increase or decrease in the amount of dispersion, thereby reducing the phase sensitivity of that particular subaperture (Fig. 8).

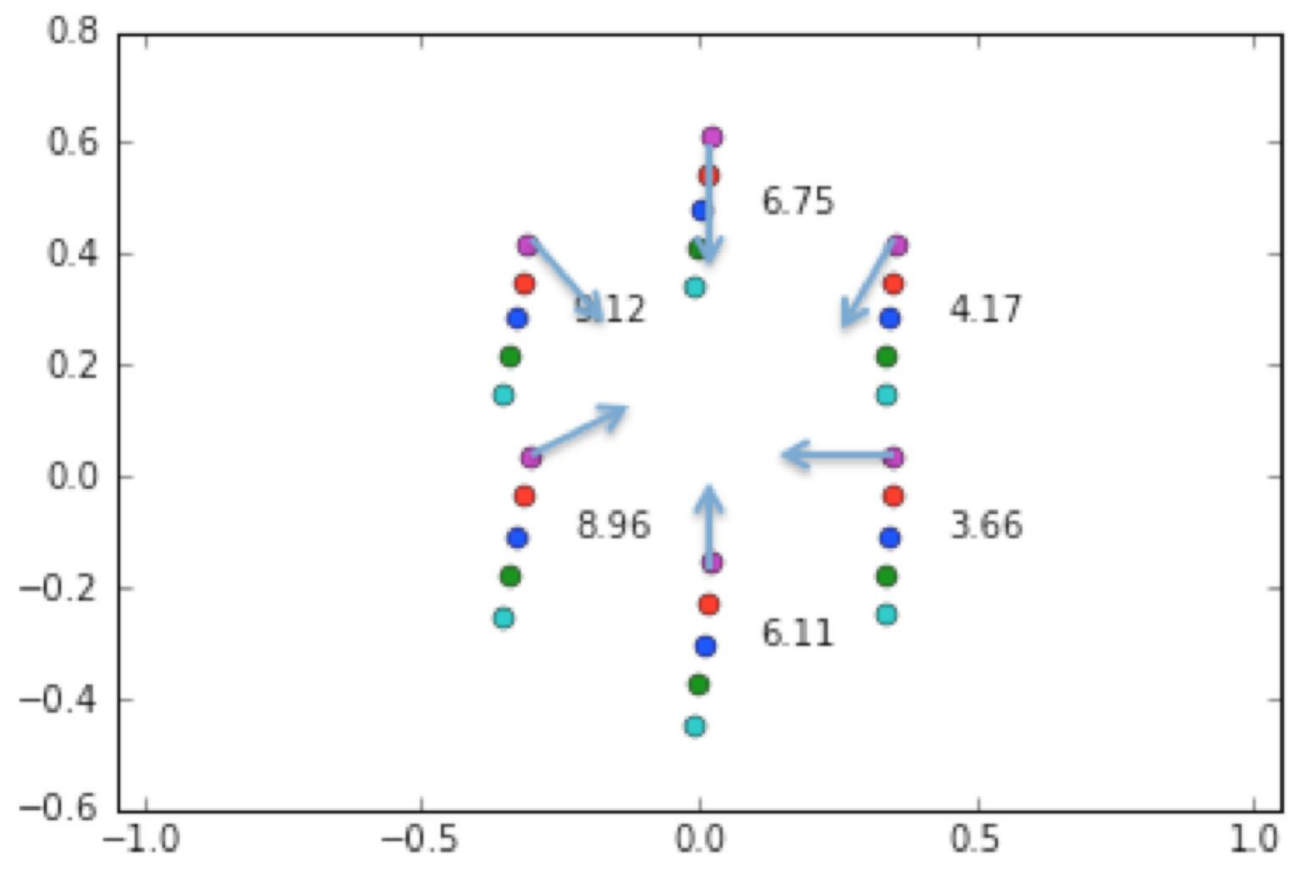

Figure 8: Fringe tilt predictions using Zemax modeling. Numbers are predicted fringe tilts in degrees. The side fringes are essentially rotated, while the top/bottom fringes are shortened/lengthened.

\section{OBSERVATIONS}

The prototype was tested on-sky for three nights, December 17-19, 2015 behind the Magellan AO system ${ }^{12,13}$ (Fig. 9). Data was taken first on-axis at both I and J bands with and without the phase shifter. The pupil viewing mode and internal tip/tilt mirror were used to align the pupil of the prototype to the telescope pupil. Various control strategies of the AO system were used to simulated both diffraction limited and seeing limited conditions. Data was taken with the 
AO system running at $1 \mathrm{kHz}$ with 300 corrected modes, with the AO system off and the adaptive secondary mirror in a static "flat" shape, and in an in-between configuration with only 10 low-order modes corrected with the intention of mitigating low-order static aberrations from the secondary and telescope, while allowing the prototype to sense the highorder atmospheric turbulence modes. On the last night, off-axis data was taken at both bands with the OAL in place.

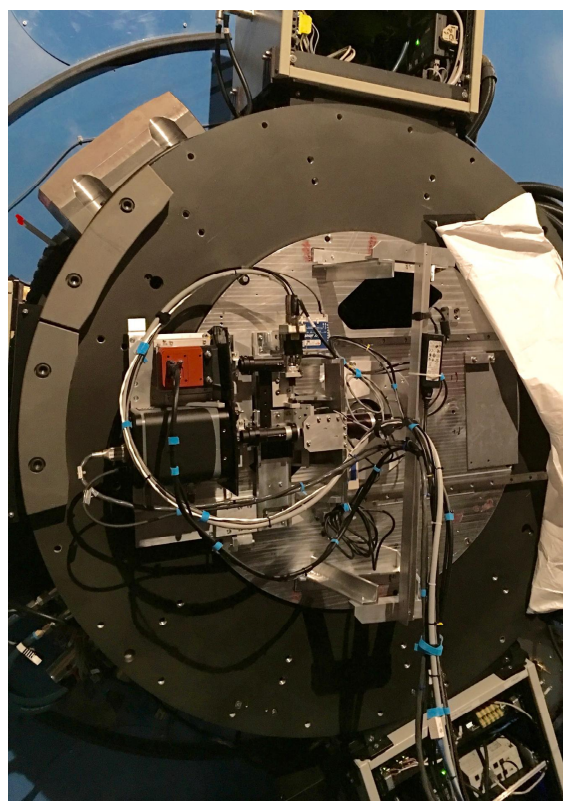

Figure 9: The SAO phasing prototype mounted on the MagAO rotator at the Magellan Clay Nasmyth platform.

\section{RESULTS}

\subsection{I-band on-axis AO corrected fringes}

Data was collected with the $\mathrm{AO}$ system running at $1 \mathrm{kHz}$ correcting 300 modes with the adaptive secondary mirror. 36000 images were captured at a frame rate of $120 \mathrm{~Hz}$ for a total of $300 \mathrm{~s}$ of data. This data was used to calculate 10 estimates of segment piston. The mean calibrated piston estimate averaged over the six subapertures is $1.25 \mu \mathrm{m}$, which is consistent with the value expected when taking into account the modeled atmospheric dispersion at the 67.8 degree elevation angle of these observations. Figure 10 shows an example of a short exposure set of fringes.
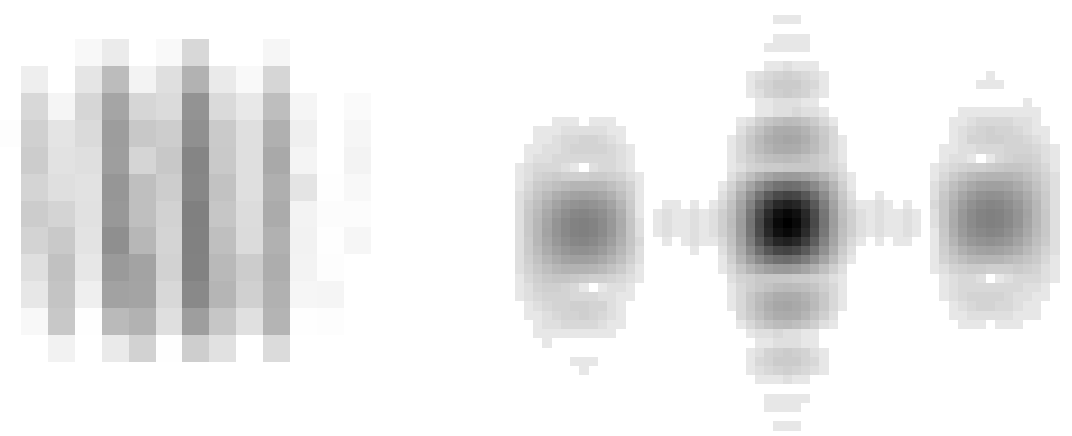

Figure 10: Left: Example of a short exposure set of fringes. Right: Absolute value of the Fourier transform averaged over 30 seconds. 


\subsection{Seeing-limited fringes}

In actual operation at the GMT, the phasing camera will need to be able to operate in seeing-limited conditions, or in conditions where the isoplanatic angle between the on-axis AO guide stars and the off-axis phasing stars is large enough for the image to be close to seeing limited. We took data with the AO loop paused, which seemed to give inconsistent results across the six subapertures, possibly from static aberrations in the secondary mirror. We then took data with the adaptive secondary in a flattened position (Fig. 11). We also took data with the $\mathrm{AO}$ system running at $1 \mathrm{kHz}$ at only 10 modes.

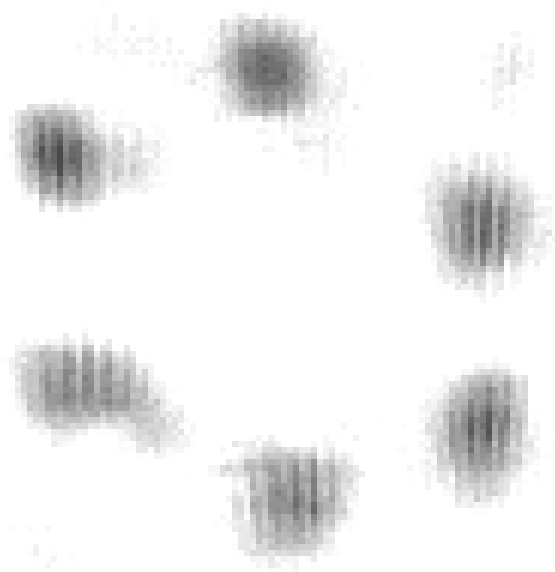

Figure 11: A typical I-band set of fringes with the AO off.

\subsection{I-band off-axis}

In science operation at the GMT, the AGWS will use phasing guide stars in a 6-10 arcmin annular FOV. One of the goals of the prototype is to determine how the DFS performs off-axis behind an AO system that is guiding on an on-axis star. Because of the complex optics required to correct the static aberrations of the Magellan telescope, which vary over the FOV, we decided to perform this test at on fixed off-axis distance of 6 arcmin.

With the prototype at 6 arcmin, we observed that turning the AO system on decreased the FWHM of the fringes, but also decreased the fringe contrast. We expect that the decrease in FWHM is the result of the AO system correcting low order aberrations, while the high order correction is poor due to isoplanatic effects, thereby resulting in the reduction in fringe contrast (Table 1). The fringe contrast is defined as the ratio between the peak of the side lobes of the Fourier amplitude with the value of the main peak.

\begin{tabular}{rrrrr}
\hline Frame & \multicolumn{2}{c}{ AO } & FWHM & \multicolumn{2}{c}{ Fringe contrast } \\
\hline 143 & 10 modes & 0.893 & 0.059 \\
\hline 144 & No AO & 0.946 & 0.056 \\
\hline 145 & 100 modes & 0.831 & 0.045 \\
\hline 146 & 100 modes & 0.863 & 0.031 \\
\hline 147 & 100 modes & 0.874 & 0.031 \\
\hline 148 & 10 modes & 0.976 & 0.050 \\
\hline
\end{tabular}

Table 1: Image quality metrics for the I-band sensor at 6 arcmin off-axis.

The phase measurements of the individual fringe sets are consistent to approximately $60 \mathrm{~nm}$ rms between the AO-on and AO-off frames, allowing us to conclude that the DFS works well off-axis.

\subsection{J-band}

Data was taken with the J-band channel both with full AO correction and with only 10 mode AO correction. Because of what are likely static aberrations in the J-band channel, only the top and bottom fringes gave good contrast for most of the exposures. $10 \mathrm{~ms}$ exposures were taken at 50 frames per second, with the camera exposing only half the time. The 
average piston value for these two subapertures when AO was on is -6.931 and -6.017 microns, with an RMS value of 0.056 microns for each measurement. These values include the biases caused by grism rotation and lateral color. Fig. 12 shows an example of the J-band fringes.

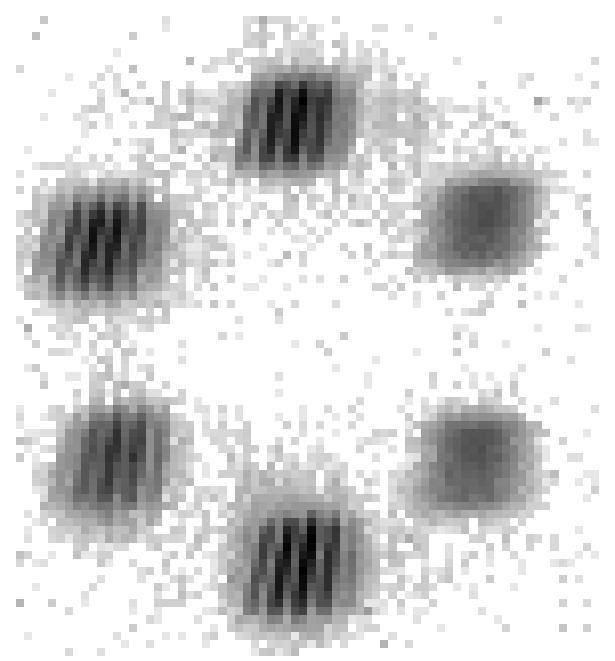

Figure 12: Example of an instantaneous $10 \mathrm{~ms}$ J-band image, on-axis, with full AO correction. Fringe on the right side, and to a lesser extent the left side, show a reduction in fringe contrast possibly due to the phase shifter or the internal optics being misaligned. The top and bottom sets of fringes were used for the analysis.

Relative to the AO-on values, the piston values for the AO-off (10 modes being corrected) data was measured to be 0.146 and 0.188 microns with RMS values of 0.262 and 0.166 microns, respectively (see Fig. 13).

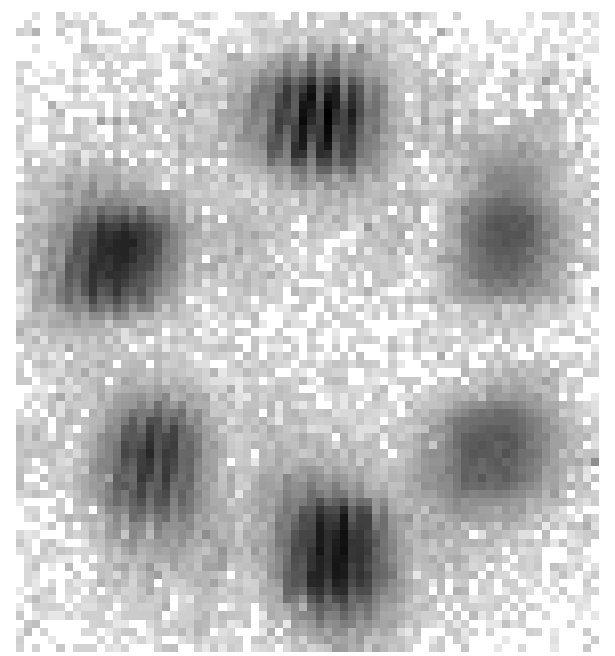

Figure 13: Example of a J-band frame, on-axis, with the AO system only correcting 10 modes and the loop gain set to 0.1 .

The capture range of the J-band sensor was measured using the phase shifter both with full AO correction and with the 10 low order correction. The sensor works out to approximately 40 microns (Fig. 14), which roughly agrees with simulations ${ }^{14}$. 


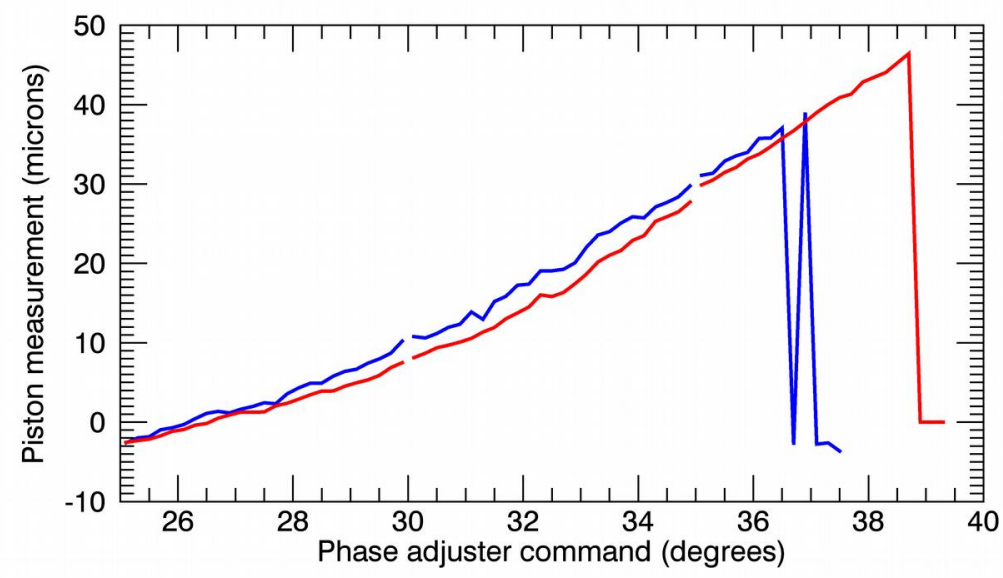

Figure 14: Piston phase difference as a function of phase shifter clocking angle for the top subaperture (blue) and bottom subaperture (red). The 40 micron cutoff range agrees with predictions.

The off-axis performance of the J-band sensor had lower contrast than expected and did not work well, most likely due to a misalignment of the stage holding the very sensitive OAL.

\subsection{Dispersed Hartmann Sensor Analysis}

With no changes in the optics or hardware setup of the prototype, the data can be reduced using a dispersed Hartmann sensor (DHS) analysis that uses Fourier phase, rather than Fourier amplitude, as in the DFS analysis. The DHS analysis is insensitive to small rotations of the dispersion direction relative to the aperture mask. The DHS analysis agrees very well with the DFS analysis after subtracting a fixed offset. This offset can be explained by the rotation of the fringes caused by the clocked grism and the lateral color, which affects the DFS measurements, but not those of the DHS (see Fig. 15).

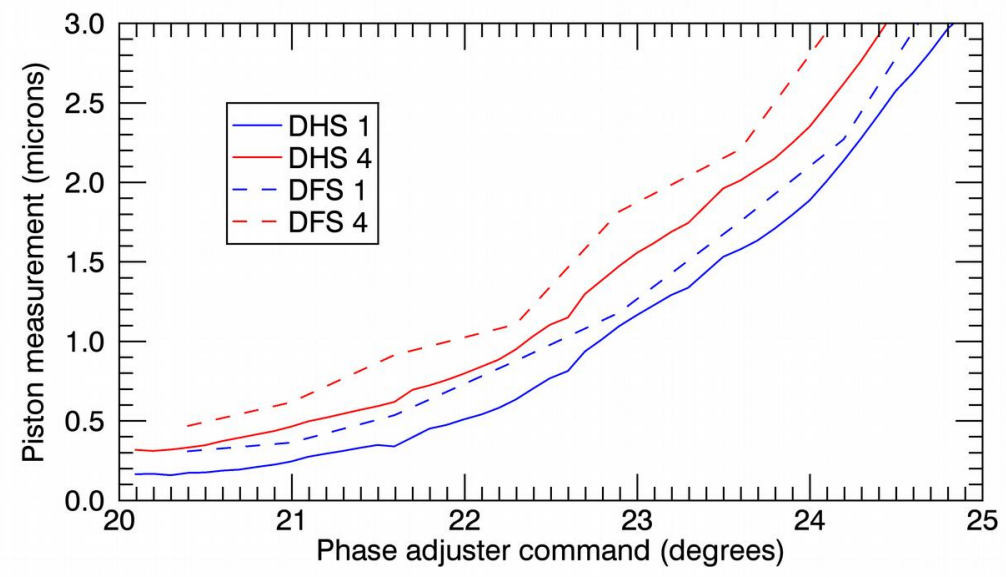

Figure 15: Comparison of the phase-unwrapped DHS and DFS results for subapertures 1 and 4. The two analyses agree very well, minus a fixed offset, which can be explained by the clocking of the dispersion direction of the fringes due to grism clocking and lateral color.

\section{CONCLUSIONS}

We present the results of three nights of observing with our prototype DFS behind the Magellan AO system. We were able to measure piston phase in both I and J bands. We noticed significant piston biases in the sensors due to the rotated grisms, lateral color in the reimaging optics, and atmospheric refraction. These biases will need to be corrected or calibrated out in future versions of the sensor. The measured rms variations in the I-band sensor $(\sim 70 \mathrm{~nm})$ were slightly 
higher than the predicted values of $\sim 50 \mathrm{~nm}$. Because the six subapertures did not all behave in the same way due to various biases, we were not able to use them to calibrate out effects such as atmospheric dispersion and grism rotation. The capture range of the J-band sensor ( 40 microns), performed as expected. The prototype worked well off-axis in Iband, but not in J-band, likely due to misalignment of the off-axis correcting optics. DHS values were extracted from the AO-corrected data and matched well with the DFS data.

\section{Acknowledgements}

This work was supported by the GMTO Corporation, a non-profit organization operated on behalf of an international consortium of universities and institutions: Astronomy Australia Ltd., the Australian National University, the Carnegie Institution for Science, Harvard University, the Korea Astronomy and Space Science Institute, The Sao Paolo Research Foundation, the Smithsonian Institution, The University of Texas at Austin, Texas A\&M University, University of Arizona, and University of Chicago. We also think the Magellan telescope staff for their help mounting and commissioning the prototype.

\section{REFERENCES}

[1] Bouchez, A. H., Acton, D. S., Biasi, R., Conan, R., Espeland, B., Esposito, S., Filgueira, J. M., Gallieni, D., McLeod, B. A., et al., "The Giant Magellan Telescope adaptive optics program," Proc. SPIE 9148, 91480W (2014).

[2] Bouchez, A. H., McLeod, B. A., Acton, D. S., Kanneganti, S., Kibblewhite, E. J., Shectman, S. A.., van Dam, M. A., "The Giant Magellan Telescope phasing system," Proc. SPIE 8447, 84473S (2012).

[3] Quirós-Pacheco, F., Conan, R., McLeod, B., Irarrazaval, B.., Bouchez, A., "Wavefront control simulations for the Giant Magellan Telescope: Field-dependent segment piston control," AO4ELT4 Conf. Proc. (2015).

[4] Albanese, M., Wirth, A., Jankevics, A., Gonsiorowski, T., Ohara, C., Shi, F., Troy, M., Chanan, G.., Acton, S., "Verification of the James Webb Space Telescope coarse phase sensor using the Keck Telescope," Proc. SPIE 6265, 62650Z (2006).

[5] Codona, J., "Pairwise Segment Phasing with the GMT" (2008).

[6] van Dam, M. A., McLeod, B. A.., Bouchez, A. H., "Dispersed fringe sensor for the Giant Magellan Telescope," Appl. Opt. 55(3), 539-547, OSA (2016).

[7] McLeod, B., Bouchez, A. H., Espeland, B., Filgueira, J., Johns, M., Norton, T. J., Ordway, M., Podgorski, W. A., Roll, J., et al., "The Giant Magellan Telescope active optics system," Proc. SPIE 9145, 91451 T (2014).

[8] Kanneganti, S., McLeod, B. A., Ordway, M. P., Roll, J. B., Shectman, S. A., Bouchez, A. H., Codona, J., Eng, R., Gauron, T. M., et al., "A prototype phasing camera for the Giant Magellan Telescope," Proc. SPIE 8447, 844710-844752 (2012).

[9] Feautrier, P., Gach, J.-L.., Wizinowich, P., "State of the art IR cameras for wavefront sensing using e-APD MCT arrays," AO4ELT4 (2015).

[10] Kopon, D., McLeod, B., McCracken, K., van Dam, M., Bouchez, A., Conder, A., Podgorski, W. A., Catropa, D., McMuldroch, S., et al., "Prototyping the GMT phasing camera with the Magellan AO system," AO4ELT4 (2015).

[11] van Dam, M., McLeod, B.., Kopon, D., "FWN 80: Laboratory measurements of the DFS prototype," internal design report (2015).

[12] Close, L. M., Males, J. R., Morzinski, K., Kopon, D., Follette, K., Rodigas, T. J., Hinz, P., Wu, Y.-L., Puglisi, A., et al., "Diffraction-limited Visible Light Images of Orion Trapezium Cluster with the Magellan Adaptive Secondary Adaptive Optics System (MagAO)," ApJ 774, 94 (2013).

[13] Morzinski, K., Close, L. M., Males, J. R., Hinz, P., Esposito, S., Riccardi, A., Briguglio, R., Follette, K., Pinna, E., et al., "MagAO: status and science," Proc. SPIE, these proceedings (2016).

[14] Quirós-Pacheco, F., Conan, R., Bouchez, A.., McLeod, B., "Performance of the Giant Magellan Telescope phasing system," Proc. SPIE 9906, 9906-246 (2016). 